\title{
Magnetohydrodynamics (MHD) axisymmetric flow and heat transfer of a hybrid nanofluid past a radially permeable stretching/shrinking sheet with Joule heating
}

\begin{abstract}
The main interest of the present work is to fundamentally investigate the flow characteristics and heat transfer of a hybrid $\mathrm{Cu}-\mathrm{Al} 2 \mathrm{O} 3 /$ water nanofluid due to a radially stretching/shrinking surface with the mutual effects of MHD, suction and Joule heating. The surface is permeable to physically allow the wall mass fluid suction. Tiwari and Das model of nanofluid is used with the new thermophysical properties of hybrid nanofluid to represent the problem. A similarity transformation is adopted to convert the governing model (PDEs) into a nonlinear set of ordinary differential equations (ODEs). A bvp4c solver in MATLAB software is employed to numerically compute the transformed system. The numerical results are discussed and graphically manifested in velocity and temperature profiles, as well as the skin friction coefficient and heat transfer rate with the pertinent values of the dimensionless parameters namely magnetic, $\mathrm{Cu}$ volume fraction, suction and Eckert number. The Eckert number has no impact on the boundary layer separation while the higher value of the suction parameter may affect the heat transfer performance. The presence of dual solutions (first and second) is seen on all the profiles within a limited range of the physical parameters. The stability analysis is executed, and it is validated that the first solution is the real solution.
\end{abstract}

Keyword: Hybrid nanofluid; Radially stretching/shrinking; Suction; Joule heating; Stability analysis 